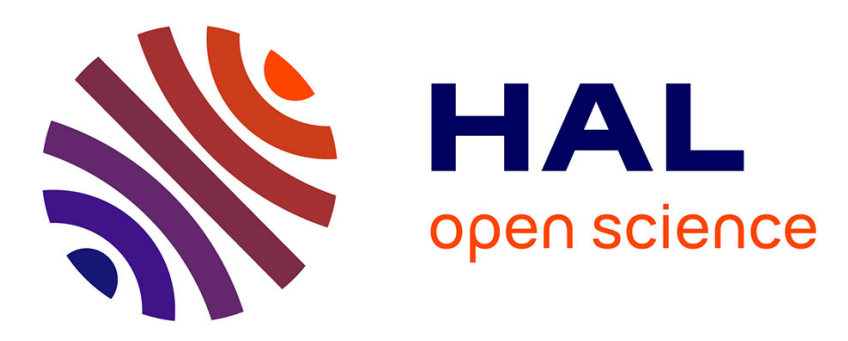

\title{
Les prises d'information dans les activités professionnelles : des éléments à prendre en compte en formation.
}

\author{
Audrey Murillo, Raquel Becerril Ortega, Bernard Fraysse
}

\section{To cite this version:}

Audrey Murillo, Raquel Becerril Ortega, Bernard Fraysse. Les prises d'information dans les activités professionnelles: des éléments à prendre en compte en formation.. Travail et apprentissages: revue de didactique professionnelle, 2011, 8, pp.30-47. halshs-00807206

\section{HAL Id: halshs-00807206 \\ https://shs.hal.science/halshs-00807206}

Submitted on 3 Apr 2013

HAL is a multi-disciplinary open access archive for the deposit and dissemination of scientific research documents, whether they are published or not. The documents may come from teaching and research institutions in France or abroad, or from public or private research centers.
L'archive ouverte pluridisciplinaire HAL, est destinée au dépôt et à la diffusion de documents scientifiques de niveau recherche, publiés ou non, émanant des établissements d'enseignement et de recherche français ou étrangers, des laboratoires publics ou privés. 


\title{
Les prises d'information dans les activités professionnelles : des éléments à prendre en compte en formation
}

\author{
Raquel Becerril, Audrey Murillo, Bernard Fraysse
}

\begin{abstract}
Murillo, A., Becerril, R., \& Fraysse, B. (2011). Les prises d'information dans les activités professionnelles : des éléments à prendre en compte en formation. Travail et apprentissages, 8, 30-47.
\end{abstract}

\section{Résumé}

Nous proposons une réflexion autour des prises d'information effectuées par les professionnels, et de l'importance de leur prise en compte dans les situations de formation. Notre propos découle de deux recherches issues de la didactique professionnelle : une recherche porte sur l'activité d'agriculteurs qui utilisent du bois broyé pour fertiliser leur sol, l'autre recherche porte sur l'utilisation d'une Machine Outil à Commande Numérique en contexte professionnel et en contexte de formation. Après avoir montré que les prises d'information des professionnels sont à la fois sources et produits de leurs représentations pour l'action, nous avançons des propositions théoriques et méthodologiques concernant l'étude des prises d'information. Nous montrons également qu'une focalisation sur les prises d'information permet d'analyser l'activité en situation professionnelle, l'activité en situation de formation, mais également l'articulation entre ces deux situations.

\section{Mots-clés}

Prises d'information, didactique professionnelle, situation professionnelle, situation de formation, Machine Outil à Commande Numérique, Bois Raméaux Fragmentés

\section{Summary}

We propose to provide lines for thought regarding gathering of information by professionals, and the importance of their inclusion in training situations. This paper results from two researches in professional didactics: the first one, focuses on the activity of farmers who use chipped ground to fertilize their soil, the second one, focuses on the use of a Computer Numerical Control Machine in professional situation and training situation. We show that information gathering is both source and product of the professionals' representations for action. We propose theoretical and methodological proposals concerning the gathering of information. Finally, we show that focusing on the gathering of information is useful to analyze the activity in professional situation, the activity in training situation, and the relationship between these two situations.

\section{Key words}

Information gathering, professional didactics, professional situation, training situation, Computer Numerical Control Machine, Ramial Chipped Wood 


\section{Introduction}

Cet article a pour ambition de porter un regard articulé sur deux recherches que les auteurs ont conduites de manière indépendante. Ces deux recherches s'appuient sur des situations professionnelles, d'une part en contexte industriel autour des Machines Outil à Commande Numérique (MOCN) et d'autre part en contexte agricole autour de la fertilisation du sol au moyen de branches et rameaux broyés.

Nous montrerons que la notion de "prises d'information » est un concept central qui permet l'articulation entre les deux recherches.

Nous interrogerons les prises d'information en tant qu'objet central de l'activité professionnelle et nous pointerons leur importance dans les situations de formation, car déterminantes dans la construction des représentations pour l'action.

Pourquoi ces prises d'information sont-elles importantes dans les activités professionnelles? Comment les mettre au jour? Pourquoi et comment les prendre en compte dans les formations professionnelles ? Pour avancer des éléments de réponse à ces questions, nous proposerons tout d'abord un cadre conceptuel permettant d'articuler les notions de situation professionnelle, de prise d'information et de représentations pour l'action, empruntées à l'ergonomie de langue française. Nous présenterons ensuite des pistes de réflexion issues de nos recherches sur les terrains industriel et agricole. Nous montrerons enfin qu'une focalisation sur les prises d'information permet d'analyser l'activité en situation professionnelle, l'activité en situation de formation, mais également l'articulation entre ces deux situations.

\section{Fondements théoriques}

\section{a. Activité professionnelle et représentations pour l'action}

Les développements de la didactique professionnelle permettent aujourd'hui d'écrire qu'une formation professionnelle pertinente se doit d'être élaborée en référence à des situations professionnelles. A l'instar de Leplat et Hoc (1983), nous entendons par situation un "système tâche-sujet ", les interactions entre la tâche et le sujet relevant de l'activité. L'analyse seule de la tâche peut conduire à une mise en évidence de savoirs et savoir-faire professionnels; ces "savoirs de la tâche " sont souvent répertoriés, organisés et énoncés (Savoyant, 2008). Toutefois, ils ne suffisent pas à rendre compte de l'ensemble des savoirs et savoir-faire professionnels mis en œuvre dans une situation; en effet, les professionnels développent aussi des "savoirs de l'activité ", "liés au contexte et aux conditions spécifiques de mise en œuvre de l'activité " (Idem). L'existence de ces savoirs de l'activité est notamment due aux écarts entre le prescrit et le réel. Ces écarts ont de multiples sources (Rabardel, Carlin, Chesnais, Lang, \& Pascal, 1998) :

- le sujet peut avoir des difficultés à se représenter et réaliser les prescriptions ;

- la prescription ne peut être exhaustive, l'activité déborde donc toujours le prescrit ;

- les situations de travail sont variables, et le sujet doit s'adapter à cette variabilité ;

- le sujet peut redéfinir son travail à partir de ses objectifs et valeurs. 
L'écart entre prescrit et réel ne constitue donc pas toujours une erreur de la part du sujet, mais il est nécessaire, et souvent lié à une adaptation aux contraintes de la situation. L'analyse des tâches ne peut donc suffire à appréhender les savoirs et savoir-faire mis en œuvre par les professionnels: c'est vers l'analyse de l'activité que nous orientons nos recherches, en nous inscrivant dans le cadre de la didactique professionnelle. L'activité professionnelle n'est pas qu'observable, elle est en grande partie cognitive, même lorsqu'il s'agit de mettre en œuvre une activité motrice. En effet, toute activité professionnelle s'appuie sur des représentations pour l'action (Weill-Fassina, Rabardel, \& Dubois, 1993), c'est-à-dire des constructions cognitives opératives. Ces représentations pour l'action ont fait l'objet de nombreuses recherches, et sont diversement nommées :

- images opératives, qu'Ochanine (1978) définit comme des "structures informationnelles spécialisées qui se forment au cours de telle ou telle action dirigée sur des objets ", par distinction avec les images cognitives, " qui sont le reflet intégral des objets dans toute la diversité de leurs propriétés accessibles »;

- représentations opérationnelles pour Vermersch (1981), qui juge le terme " image » trop restrictif, et préfère l'adjectif "opérationnel » à " opératoire " pour décrire le caractère adapté de la représentation, tout en évitant la confusion avec la « pensée opératoire " piagétienne ;

- représentations fonctionnelles pour Leplat (1985), dans le sens où elles sont immédiatement exploitées à une fin autre que contemplative, notamment dans des situations professionnelles.

\section{b. Représentations pour l'action et prises d'information}

Comme le précisent Weill-Fassina, Rabardel et Dubois (1993, p.17), les représentations pour l'action sont à la fois des " processus actifs de prise de connaissance et d'appropriation des situations " et des résultats de l'activité des individus. Ainsi, la situation-« arena " (Lave, 1988) est une donnée objective partiellement exploitée par le professionnel, notamment au moyen de prises d'information. Les interactions entre le sujet et la situation-« arena » permettent la construction de représentations pour l'action, et les prises d'information sont médiatrices dans la construction des représentations pour l'action.

Par la construction de ces représentations, la situation-« arena » devient une situation" setting " (Idem) : les prises d'information, guidées par les représentations, permettent une appréhension opératoire de la situation. 


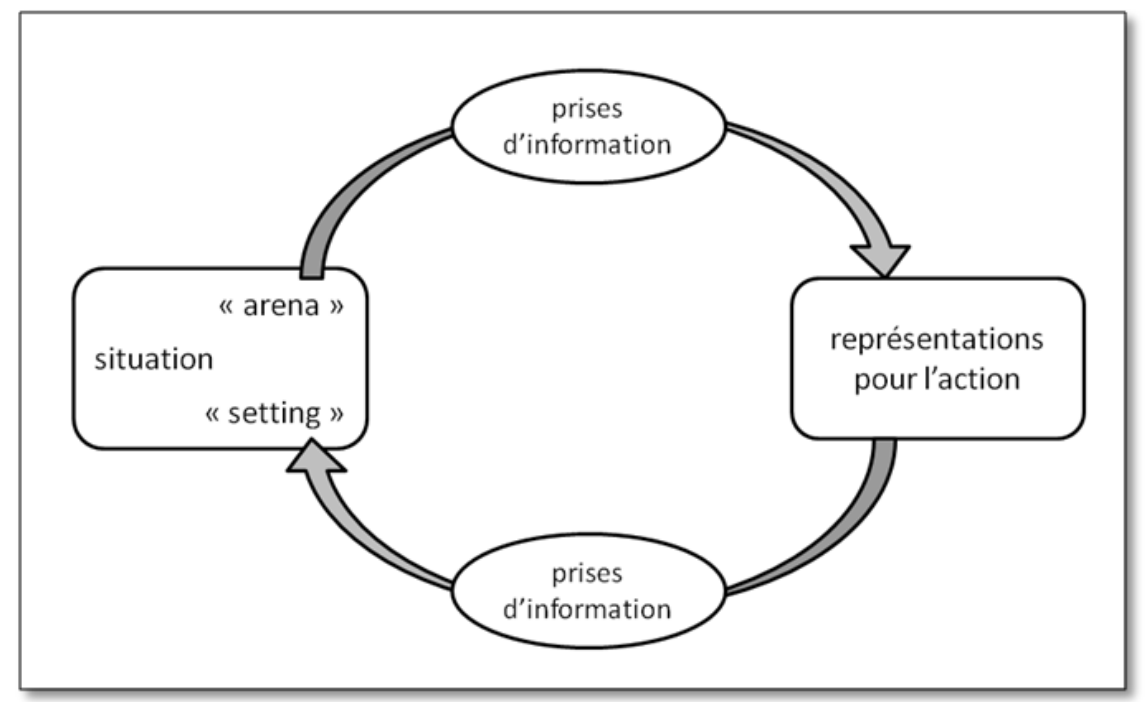

Les prises d'information permettent la construction des représentations pour l'action, et en sont des produits

Ainsi, les professionnels ne sont pas de simples "récepteurs " d'informations (visuelles, auditives, tactiles...), mais bien aussi des "fureteurs" (Desnoyers, 2004)... même si le prélèvement d'informations n'est pas toujours réfléchi et conscient (Vergnaud, 1985).

Les prises d'information étant à la fois sources et produits des représentations pour l'action, les caractéristiques des représentations pour l'action peuvent nous renseigner, au moins partiellement, sur celles des prises d'information. Les caractéristiques des représentations pour l'action sont les suivantes.

- Elles sont fonctionnelles, dans la mesure où elles « assure[nt] la planification et le guidage de l'action » (Leplat, 1985). Elles ne sont donc pas " contemplatives » (Idem), destinées à décrire un objet sans aucune autre fin que sa caractérisation. Notons que la fonctionnalité préjuge de la fonction à remplir, mais pas de la réussite dans la tâche (Teiger, 1993).

- Elles sont opératives, c'est-à-dire propres à produire des effets. Le degré d'efficacité des représentations opératives peut toutefois varier (Idem). Les représentations opératives sont opérationnelles « lorsqu'elles offrent à l'opérateur le moyen de planifier et/ou de guider son action de manière adéquate à la situation actuelle " (Bertrand \& Weill-Fassina, 1993).

- Elles dépendent de la tâche à effectuer. Ainsi, les anesthésistes et les infirmiers ne portent pas leur attention sur les mêmes informations d'une fiche d'anesthésie (Thuilliez, 2005), tout comme les physiologistes et les bouchers ne décrivent pas identiquement un muscle d'animal (Beauvois \& Dubois, 1991)

- Elles se construisent avec l'expérience. Pour les débutants, les représentations sont faiblement opérationnelles. C'est le cas des professionnels ayant à diagnostiquer des pannes (Bertrand \& Weill-Fassina, 1993), ou des enseignants (Carter, Cushing, Sabers, Stein, \& Berliner, 1988) : des commentaires de photographies de classe réalisés par des enseignants novices et expérimentés diffèrent par l'utilité des informations prises en compte. Par exemple, un enseignant débutant mentionne la couleur des cheveux des élèves, tandis qu'un expérimenté repère leurs positions dans la classe, et en infére un travail de groupe. Les travaux de Chase et Simon (1973) ont également montré que les 
joueurs d'échec expérimentés étaient mieux à même de mémoriser des positions de pièces car ils agrégeaient les pièces en unités plus larges faisant sens pour eux (des pièces positionnées au hasard sur l'échiquier et ne correspondant pas à une partie réelle n'étaient pas mieux mémorisées par les joueurs expérimentés).

- Elles sont caractérisées par leur pertinence plus que leur véracité (Weill-Fassina et al., 1993 ; Leplat, 1985). Diverses représentations d'un même objet peuvent être vraies, mais les plus opérationnelles sont les plus pertinentes, et c'est sur ce critère qu'elles peuvent être distinguées. Les représentations qu'ont les enseignants débutants d'une photographie de classe (Carter et al., 1988) ne sont pas fausses lorsqu'ils mentionnent la couleur des cheveux des élèves; elles sont toutefois peu pertinentes.

- Elles sont sélectives. Dans la situation, tout ne peut pas, et ne doit pas, être l'objet d'une prise d'information. Ochanine (1978) évoque ainsi le "laconisme " des images opératives: à l'inverse des images cognitives, elles ne prennent en compte que les propriétés utiles pour accomplir la tâche. Ceci est nécessaire pour des raisons d'économie cognitive, mais également, selon Rabardel (1993), pour laisser du " jeu » permettant l'adaptation à des situations singulières.

- Elles sont déformantes (Leplat, 1985). En plus d'ignorer certaines propriétés des objets, elles en surestiment certaines et en sous-estiment d'autres. Par exemple, lorsqu'il est demandé à des médecins expérimentés de dessiner une thyroïde, ces derniers grossissent les parties les plus importantes pour le diagnostic (Ochanine, 1978). Enard (1968) montre également que les contrôleurs aériens survalorisent dans leur " carte géographique mentale » les points nécessitant le plus d'attention. Similairement, les utilisateurs d'un centre de documentation amenés à dessiner un plan de ce centre mettent en exergue les endroits, et même les objets, qu'ils utilisent le plus souvent (Fabre \& Veyrac, 2008).

\section{Problématique et résultats de recherche}

Notre approche s'intéresse aux prises d'information comme notion permettant une lecture à la fois de l'activité professionnelle et de l'activité de formation professionnelle. Nous interrogeons donc les prises d'information des acteurs dans chacun des contextes, professionnel et de formation. L'étude que nous développons s'intéresse d'abord aux situations professionnelles. Dans ce cadre, nous identifions les prises d'information de professionnels de l'agriculture et de l'industrie.

Dans le contexte agricole, nous identifions les prises d'information des professionnels en situation de travail. Dans le contexte industriel, nous identifions également les prises d'information des apprenants en situation de formation. Nous prenons en compte d'une part le fait que les situations de formation professionnelle s'inspirent de situations professionnelles de référence, en termes de légitimité et de pertinence (Rogalski \& Samurçay, 1994), et d'autre part que les prises d'information dépendent des choix de l'enseignant et des effets de la transposition des situations (par exemple, une classe n'est pas un atelier).

A partir de la notion de prises d'information, nous approchons la pertinence des situations de formation professionnelle proposées : la prise d'information étant un élément important de construction des représentations pour l'action, nous en inférons son 
importance dans le processus de construction de compétences professionnelles en situation de formation.

Les résultats présentés ici sont issus de deux études :

- I'une porte sur l'activité d'agriculteurs fertilisant leurs terres au moyen de branches et rameaux broyés (Murillo, 2010 ; Fraysse \& Murillo, 2010) ;

- l'autre porte sur l'activité de professionnels et d'apprenants utilisant une machine outil à commande numérique (Becerril, 2008).

\section{a) Etude de l'activité d'agriculteurs utilisant des Bois Raméaux Fragmentés}

L'activité professionnelle analysée dans cette étude est celle d'agriculteurs utilisant des "Bois Raméaux Fragmentés " (BRF) pour fertiliser les sols qu'ils cultivent. Les BRF sont un broyat de jeunes branches et rameaux d'arbres (majoritairement des feuillus). La mise en paillis de BRF ou son incorporation dans la couche supérieure du sol favorise la création d'humus et la mobilisation des minéraux nécessaires à la croissance des cultures. Ce matériau est ainsi considéré comme un aggradant (à l'inverse d'un dégradant) et présente une solution pour restaurer les sols épuisés. La procédure d'utilisation des BRF se décline en quatre étapes: coupe des branches, broyage des branches, épandage du broyat sur le sol, incorporation éventuelle du broyat au sol. Nous avons réalisé des observations et des entretiens d'autoconfrontation auprès de deux agriculteurs expérimentés et un débutant. Cette étude nous permet d'avancer des propositions théoriques méthodologiques concernant les prises d'information.

\section{par les professionnels}

* Certains buts des prises d'information ne peuvent être mis en exergue que

L'étude des prises d'information pose des questions méthodologiques. II s'agit de repérer le moment de la prise d'information, les éléments pris en compte, mais également les buts de cette prise d'information. Ces buts peuvent être inférés par le chercheur, mais il arrive que seuls les professionnels soient à même d'en rendre compte. Des entretiens avec les professionnels sont donc nécessaires pour comprendre les raisons de certaines prises d'information.

Ceci peut être illustré par l'analyse de l'activité d'un professionnel produisant et utilisant des Bois Raméaux Fragmentés. Un même maraîcher, à deux reprises, sent une poignée de terre. A ces deux moments, la question posée par le chercheur est la même : "Qu'est-ce que vous sentez ? ". Les réponses diffèrent. Dans un premier cas, le professionnel explique que, la terre étant "un peu grise ", il souhaite s'assurer qu'elle ne soit pas " un tout petit peu asphyxiée ". Dans le second cas, il cherche à savoir "si ça sent le champignon ", indicateur du degré de fertilité du sol. Comme l'odeur de champignon n'est pas encore perceptible, le maraîcher en infère que "c'est du travail en cours ". II ne suffit donc pas pour le chercheur de repérer que le professionnel opère une prise d'information. Des entretiens doivent permettre d'en déterminer l'utilité.

Si les buts des prises d'information peuvent être dans un premier temps obscurs pour le chercheur, la nature même des prises d'information peut l'être aussi. 


\section{* La nature de certaines prises d'information ne peut être mise en exergue que par les professionnels}

Dans certains cas, comme dans les exemples ci-dessus, le chercheur repère qu'il y a une prise d'information de la part du professionnel, et demande à ce dernier de l'expliciter. Le chercheur peut repérer le ou les sens mis en activité. Il s'agit dans les illustrations précédentes de l'odorat. II peut aussi s'agir de la vue : par exemple, lorsque le maraîcher examine le sol en s'accroupissant. II s'agit alors de déterminer si le BRF peut être incorporé au sol, ou s'il faut attendre quelques jours: "Les vers de terre et puis 3 ou 4 espèces de cette microfaune $m^{\prime}$ indiquent que je peux commencer à envisager [...] je vais pouvoir incorporer ". Le chercheur repère qu'il y a observation, et le professionnel explicite la nature de ce qui est observé (ainsi que les inférences qu'il effectue).

Dans d'autres cas, le chercheur peut ne pas être conscient de certaines prises d'information. A titre d'exemple, nous avons observé un professionnel en train de broyer des branches, et faisant face à un bourrage du broyeur. II est important de noter que le professionnel porte un casque de sécurité avec protection auditive et visière. Lorsque le professionnel essaie de débourrer le broyeur, il enlève son casque. Selon le chercheur, il peut s'agir soit d'une question de confort (hypothèse : le casque tient chaud et est inutile tant que des branches ne sont pas insérées dans le broyeur) ou soit d'une question de gêne pour observer la machine (hypothèse : enlever la visière permet de mieux observer l'endroit du bourrage). Lors de l'entretien d'autoconfrontation, le professionnel explicite son activité : il s'agissait en fait d'ôter la protection auditive pour mieux entendre le bruit de la machine ("j'ai enlevé le casque parce que je jugeais mal, par rapport au bruit que ça fait »).

La nature même des prises d'information, en plus de ses buts, n'est donc parfois pas accessible par une analyse uniquement extrinsèque de l'activité. Par conséquent, il est nécessaire de demander au professionnel d'expliciter cette activité. Toutefois, le discours des professionnels a ses limites, comme nous allons le montrer ci-après.

\section{toujours de décrire de façon pertinente leurs prises d'information}

* Les signifiés du discours des professionnels ne permettent pas

L'analyse des prises d'information peut difficilement se limiter au seul discours des professionnels. En effet, ces derniers ne sont pas toujours en mesure d'expliciter précisément ces prises d'information (Maurice, 2006). Par exemple, lorsqu'un professionnel produit du broyat, il précise que le résultat est " idéal ", " agréable », " cohérent "... or, ces adjectifs ne disent rien des caractéristiques qui font que le broyat est idéal, agréable, beau... Une explicitation plus poussée est alors nécessaire. Nous avons ici demandé au professionnel de commenter des photographies de différents broyats pour aider l'explicitation. Nous parvenons ainsi à extraire des caractéristiques objectives sur les informations prises par le professionnel: grosseur des morceaux, présence de sciure, mélange des branches et rameaux, sens de la coupe (dans la longueur ou la largeur de la branche).

Cette objectivation des prises d'information semble essentielle lorsqu'on s'intéresse à la formation professionnelle. Comment fixer les objectifs à atteindre, comment indiquer les erreurs, comment évaluer, si les critères ne sont pas formalisés ? Communiquer, former, incite à mettre en mots ce qui n'est pas habituellement verbalisé. Par exemple, un maraîcher observé, tout en émiettant une motte de terre, évoque le terme de "couscous" pour décrire la texture de la terre qui a reçu du BRF : "En faisant ça, [on sent] que ça y est c'est 
parti, c'est en train d'évoluer. Parce qu'on sent... on voit qu'on est proche de ce que Claude Bourguignon appelle le couscous, c'est-à-dire les petites boulettes rondes qui roulent sous les doigts. Là on est tout près de ça. " C'est le besoin de communiquer qui a conduit à verbaliser ce concept-en-acte (Vergnaud, 1985) : trouver des mots signifiants est nécessaire pour la formation. Ici, il s'agit d'un concept pragmatique (Pastré, 2002), non scientifique, mais explicite et pertinent.

\section{être fortement contextualisées}

* Les inférences réalisées à partir des prises d'information peuvent

Les prises d'information permettent de réaliser des inférences. Nous avons analysé l'activité d'un professionnel qui abat un arbre pour en couper les branches, dont certaines sont destinées à la production de Bois Raméaux Fragmentés. L'arbre tombé, le professionnel se dirige vers le tronc et observe la sève. Il en infère que la montée de sève a débuté. S'ensuit une seconde inférence du professionnel : "C'est peut-être un peu trop tard». En effet, une règle suivie par certains utilisateurs de BRF est que les branches doivent provenir d'arbres "en dormance ". Toutefois, le professionnel module cette règle générale par des éléments de contexte : d'une part, le BRF sera de qualité car il provient d'un chêne, d'autre part, la parcelle destinée à recevoir le BRF en avait déjà reçu quelques années auparavant ${ }^{1}$. La coupe tardive aura donc peu d'effets sur l'efficacité de la procédure.

Dans le domaine agricole, les situations sont généralement dynamiques ${ }^{2}$, et les règles générales sont ainsi modulées par divers paramètres pris par les situations: climat, maladies, adventices, etc. Comme nous venons de l'illustrer, une même prise d'information ne conduit pas toujours à la même inférence, il est donc malaisé de répertorier des règles générales valables quelles que soient les conditions. La connaissance du contexte local joue un rôle important dans les prises de décision. Pour concevoir une situation de formation, un certain degré de généralisation et de standardisation est souvent nécessaire. Toute la difficulté consiste donc pour les formés à apprendre des invariances, tout en les modulant par les différents paramètres pris par les situations rencontrées.

\section{* Prendre la bonne information n'est pas suffisant}

Discerner les informations pertinentes est nécessaire au bon déroulement de l'activité. Ces informations doivent également mener à des inférences pertinentes. Par exemple, constater que diverses espèces de microfaune sont présentes dans le sol ne suffit pas : il faut en tirer des indications sur la fertilité du sol, et déterminer ainsi si le sol est prêt à recevoir l'incorporation de BRF.

Or, le processus [prise d'information -> inférence $->$ choix de conduite] n'est pas toujours suffisant pour mettre en œuvre une activité optimale, notamment dans le cas de pannes, où il s'agit d'interroger le dispositif plus que de le transformer (Bertrand \& WeillFassina, 1993). Un dysfonctionnement fréquemment rencontré par les professionnels produisant des BRF est le bourrage du broyeur. Nous avons observé deux professionnels, un

1 Extrait d'entretien : «Le sol (...) reconnaît ce que je lui amène, donc ça pose pas le problème (...) qui se pose quand on est en première année, où le sol fonctionne sur un mode agricole et reconnaît pas forcément la nourriture qu'on lui emmène, qui est plutôt de type forestier. »

2 C'est-à-dire qu'elles « évoluent au cours du temps en l'absence d'action de l'opérateur » (Samurçay \& Hoc, 1988). 
novice et un expérimenté, face à un tel problème. Tous deux repèrent l'incident au moyen de prises d'information identiques : d'une part, les branches insérées ne sont plus entraînées par la machine, d'autre part, le broyat n'est plus éjecté. Novice et expert résolvent l'incident de la même manière : ils procèdent au débourrage au moyen d'une longue branche droite. Dans les deux cas, le problème est résolu. Une différence existe toutefois. Le professionnel expérimenté effectue un diagnostic et remonte à la cause de la panne: il se rend compte que la dernière branche qu'il a insérée était trop tordue, et a dû provoquer le bourrage. Le professionnel débutant, quant à lui, émet plusieurs hypothèses explicatives concurrentes quant à la cause du bourrage : mauvais réglage de la vitesse du rotor, défaut de la machine (qui était tombée précédemment), branches insérées trop rapidement... Ce défaut de diagnostic par le novice a pour conséquence que ce dernier est dans l'incapacité de prévenir et éviter un prochain bourrage du broyeur.

Ainsi, la prise d'information n'est pas utile en soi, mais l'est d'une part si elle permet de faire des choix de conduite, et d'autre part si elle est source d'un diagnostic (lorsque ce dernier est nécessaire).

b) Etude de l'activité de professionnels et d'apprenants utilisant une machine outil à commande numérique

Les résultats présentés dans cette partie proviennent d'études de cas concernant l'activité d'opérateurs sur une Machine Outil à Commande Numérique ${ }^{3}$ enlevant de la matière sur une pièce, en contexte industriel et de formation.

\section{* L'accent mis sur certaines prises d'information peut être une aide pour certains professionnels, et un obstacle pour d'autres}

En début d'activité, les opérateurs doivent régler la machine pour la préparer à un processus d'usinage automatisé. Cette procédure de réglage constitue pour les novices à la fois un obstacle épistémologique et un obstacle didactique ${ }^{4}$ :

- Le réglage de la machine constitue un obstacle épistémologique : il s'agit de concevoir l'indépendance entre la pièce à usiner et le repère en trois dimensions dans lequel son mouvement s'inscrit. Un chef d'atelier expert précise que les novices ont des difficultés à concevoir la rotation des repères: " [...] on tourne la pièce mais le repère tourne avec la pièce évidemment et beaucoup de gens ont du mal, les gens tournent la pièce mais ils ne tournent pas les repères [...]. Les gros problèmes avec les bac pro c'est la rotation des repères et des pièces " (Becerril, 2008). Ainsi, si le repère n'est pas vu comme dépendant de la pièce, les prises d'information sur la pièce sont erronées. Ces difficultés peuvent être accentuées par le type d'enseignement du dessin technique dans le cadre de l'enseignement professionnel. En effet, comme le notent Rabardel, Rak et Vérillon (1988), cet enseignement

$3 \quad$ Une machine outil est utilisée comme moyen de production dans le contexte industriel pour réaliser l'enlèvement de matière (tailler, découper, déformer de matériau). Quand elle possède une commande numérique, on parle de Machine Outil à Commande Numérique (MOCN). La Machine Outil à Commande Numérique dont il est question dans cette étude est un centre d'usinage du type $\mathrm{NUM}^{\circledR}$ (marque du centre d'usinage).

$4 \quad$ Les obstacles épistémologiques dépendent intrinsèquement de la connaissance, tandis que les obstacles didactiques sont «ceux qui semblent ne dépendre que d'un choix ou d'un projet du système éducatif » (Brousseau, 1998, p.125). 
privilégie les apprentissages de type procédural, en valorisant la forme du savoir au détriment de son « sens".

- Le réglage de la machine donne également lieu à un obstacle didactique. Les Machines Outils à Commande Numérique ont remplacé les machines outils manuelles dans les années 1980. Ceci a entraîné un bouleversement dans l'activité des opérateurs peu qualifiés. Ces derniers ont notamment rencontré une difficulté majeure dans le calcul des vecteurs impliqués dans la position relative des parties mobiles et non mobiles de la machine, désormais numérique. Pour dépasser cet obstacle, les constructeurs des machines ont introduit un intermédiaire, "l'origine pièce", point physique pour lier deux vecteurs concernés dans la mise en œuvre. Cet intermédiaire constitue donc une tentative anthropocentrée (Rabardel, 1995) de prendre en compte les compétences des opérateurs de l'époque dans la construction de nouvelles machines automatisées (c'était aussi plus économique). Il s'agit donc d'une ingénierie didactique destinée à dépasser un obstacle épistémologique, et faisant en sorte que les prises d'information pertinentes lors de l'utilisation de la machine conventionnelle le soient toujours lors de l'utilisation de la MOCN. Or, aujourd'hui, les opérateurs issus d'une formation BTS $^{5}$ en productique mécanique ne sont pas confrontés aux mêmes difficultés que leurs aînés moins qualifiés, car ils ont reçu des enseignements sur la vision vectorielle dans l'espace. Comme nous avons pu l'observer auprès de six étudiants en formation (Becerril, 2008), l'ingénierie didactique qui a facilité l'activité des opérateurs dans les années 1980 devient alors un obstacle didactique en formation initiale, et ce en BTS davantage qu'en bac professionnel de la même orientation.

\section{* La différence des informations mises à disposition en situation professionnelle et en situation de formation peut conduire à des modèles opératifs distincts}

En situation professionnelle de réglage de la machine, lorsque des novices sont confrontés à l'obstacle du positionnement du repère par rapport à la pièce, il leur est demandé d'observer un cube auquel on "attache " des repères, afin de leur montrer que le repère tourne en même temps que le cube. Le chef d'atelier exprime cette solution: "Donc on a souvent des cubes, avec des repères $x$ y $z$ et on leur montre, voyez quand on tourne. Alors ça c'est le problème qu'ils ont ». Dans la situation professionnelle, l'obstacle rencontré par les novices est ainsi résolu par une situation ("faire un cube») leur permettant une représentation opérative et fonctionnelle.

Cet exemple permet d'illustrer la problématique des prises d'information en situation professionnelle et de formation. Dans la construction de la représentation de la situation, nous avons recensé le rôle de l' "origine pièce ", un point physique de la machine qui permet de matérialiser un mouvement relatif entre les axes mobiles. Lors de nos observations de situations professionnelles et de formation, nous avons constaté des différences dans l'utilisation des termes employés et des informations à prendre. Par exemple, en situation de formation, pour calculer un vecteur, l'enseignant demande aux étudiants d'utiliser l'origine pièce, l'origine programme et la longueur d'outil. Quant à l'opérateur, il n'évoque pas l'origine programme. Ce point se retrouve dans la simplification effectuée dans le manuel d'utilisation pour les professionnels, lors du passage de l'expression vectorielle à une expression algébrique. Rabardel, Rak et Vérillon (1988)

Brevet de technicien supérieur. 
présentent les réductions effectuées dans les expressions vectorielles et l'approche procédurale proposée par certains manuels; ces auteurs indiquent qu'elles se prêtent peu à "la constitution par l'opérateur d'une conceptualisation globale du problème ", "sont spécifiques d'une machine (ou d'un constructeur)", "permettent difficilement la récupération d'incidents inhabituels ", et " préparent mal les professionnels aux évolutions des matériels ainsi que de leurs modes d'information et de gestion ".

En fonction des informations mises à la disposition des professionnels, la représentation de la situation de réglage de la machine diffère. Chez l'opérateur, la prise d'information permet la construction d'une représentation permettant d'être efficace dans un certain nombre de situations, mais lorsque les plans d'usinage se complexifient et que les repères changent en cours d'usinage, la capacité d'action s'affaiblit. Concernant les apprenants en situation de formation, s'agissant de futurs techniciens supérieurs, qui seront des concepteurs de pièces ou de programmateurs, les enseignants tentent de permettre aux apprenants de construire une conceptualisation globale du système. Alors que les techniciens usinent la pièce, les étudiants de BTS seront amenés à la concevoir : leurs activités ne sont pas de même nature et les informations mises à disposition par la situation diffèrent.

\section{* En situation de formation, des prises d'information nécessaires en situation professionnelle peuvent être occultées}

En situation professionnelle, lorsque la température extérieure est élevée ou que la machine fonctionne depuis longtemps, les éléments de la machine outil chauffent et se dilatent. C'est notamment le cas de la broche, élément de la machine qui permet l'enlèvement de matière. La dilatation de la broche est intimement liée à la qualité de la pièce usinée. Une modélisation permet de calculer la variation de la longueur de la broche en fonction du temps et des conditions de coupe. Lors de l'enlèvement de matière d'une pièce, la dilatation de la broche provoque un décalage dans la valeur de la finition. Pour corriger ce décalage, l'opérateur observe et mesure le décalage dans la pièce avant la finition, et corrige la côte ou change la longueur de l'outil. L'information se prélève de manière environnementale (bruit de la broche, chaleur de la salle), visuelle et gestuelle (observation et mesure de la pièce à I'aide d'instruments). Elle est essentielle pour guider efficacement l'activité de l'opérateur.

En situation de formation, la dilatation de la broche est présentée aux apprenants, par le biais d'un simulateur informatique, comme étant un phénomène sur lequel ils n'ont aucune prise. La modélisation effectuée dans le simulateur affiche selon une échelle de couleurs les différentes profondeurs dans la pièce, conséquences de la dilatation de la broche. Nous avons interrogé l'enseignant concepteur de la situation de formation, qui évoque les raisons de ce choix: "c'est d'abord parce qu'on ne sait pas comment la broche se dilate. Ensuite, c'est parce qu'il semble plus facile d'afficher les défauts sur la pièce. " Les informations apportées aux apprenants permettent de les sensibiliser au sujet des pièces de différentes profondeurs d'usinage; ils peuvent donc construire une représentation du résultat final, mais en aucun cas des représentations d'action leur permettant d'agir pour compenser la dilatation de la broche. 


\section{Discussion}

Nous proposons ici de soumettre à la discussion, en référence aux contextes agricole et industriel, d'une part la question de l'instrument en lien avec la prise d'information et d'autre part la question des modalités de référencement des prises d'information; ces deux questions étant mises en jeu tant dans la situation professionnelle que dans la situation de formation.

\section{a) L'instrument peut tenir l'information à distance}

Les deux agriculteurs expérimentés que nous avons observés évoquent une distance méfiante envers les machines, notamment le tracteur. Ceci s'explique en partie par un souhait d'utiliser au minimum les énergies fossiles, mais également par le fait que les machines empêchent les prises d'information : " c'est sûr qu'en travaillant comme ça [en épandant le broyat à la brouette], même si ça prend beaucoup plus de temps, ce qu'on met sur le sol, on voit ce qu'on y met, et on voit dans quel état est le sol aussi. (...) Quand on fait ça, ben on n'est pas loin du sol, donc on voit bien comment le sol est. " Alors que les machines facilitent le travail et peuvent devenir des instruments pour les professionnels, peut se poser un problème de transparence opérative (Rabardel, 1995): la machine " éloigne " du sol, du broyat, et de possibles prises d'information.

Similairement, un professionnel débutant explique qu'il fait faire des analyses de sols tous les ans, ce qui lui permet d'avoir des données techniques précises sur l'état de ses terres. Ces prises d'information extrêmement précises se font en dépit de prises d'information non instrumentées mais régulières, prônées par un maraîcher expérimenté : "[Je fais] de l'observation très régulièrement sur les endroits où on a emmené du broyat, notamment pour choisir le moment où on va faire l'incorporation (...) et je pense que dans l'appropriation de la technique et de l'itinéraire, ça permet de bien sentir le sol sur lequel on va mettre la couche [de broyat] (...). Mais c'est plus du domaine du senti que du domaine de critères techniques. " L'utilité de ces prises d'information n'est pas systématique, mais elle offre au professionnel l'opportunité de mieux connaître son contexte de travail.

En ce qui concerne le contexte industriel, les opérateurs expérimentés, ayant une expérience dans le domaine des machines outils conventionnelles (sans commande numérique), évoquent comment ils " réinvestissent les compétences issues de la conduite de la machine-outil manuelle " (Samurçay et Rabardel, 2004, p 167) : "Ça vous permet de voir l'usinage autrement que sur un centre, et c'est là que si vous savez bien usiner dans le conventionnel, après sur un centre [numérique] c'est facile, parce qu'on a des combines, qu'après sur un centre, celui qui n'a jamais fait de conventionnel ne sait pas ». Concernant les prises d'information, ils évoquent certaines différences par rapport aux machines outils à commande numérique. Tout d'abord, les prises d'information peuvent se faire en manuel tout au long du processus alors qu'en commande numérique on doit attendre la fin du processus. "En conventionnel, oui, on le sent. Dans le centre non, ou des fois c'est trop tard ". D'autres différences quant à la prise d'information ont été évoquées: "Oui, [la machine conventionnelle] est plus sensible, on le sent davantage, et puis de toute façon c'est plus facile, pas plus facile mais questions de coupe, on l'ouvre on le fait manuellement, on 
regarde, ce que ça fait, et quand on est bon on met l'avance et puis voilà. Bon, on peut le faire pareil au centre d'usinage mais c'est pas pareil mais déjà, on est plus loin, on ne voit pas bien, il faut fermer une porte, c'est pas pareil, c'est pas du tout pareil ". En effet, dans la MOCN les prises d'information s'effectuent en dehors de la machine qui est une " ressource mobilisée ou mobilisable pour l'activité productive du sujet » (Rabardel, 2005, p.14).

\section{b) Comment prendre en compte les prises d'information en situation de formation ?}

Nous avons montré que les prises d'information jouent un rôle important dans les situations professionnelles: rôle dans la construction des représentations pour l'action, et dans l'action efficace. Or, en situation de formation, deux difficultés apparaissent :

- d'une part, le formateur n'est pas nécessairement conscient des prises d'information effectuées par les professionnels ;

- d'autre part, il est plus aisé pour le formateur de se centrer sur les productions des apprenants, leur comportement, que sur leur activité cognitive (Pastré, Mayen \& Vergnaud, 2006 ; Murillo, 2010) : les informations que prennent les apprenants sont difficiles d'accès pour le formateur.

L'explicitation des prises d'information effectuées par les professionnels est-elle souhaitable? De nombreux professionnels ont construit leurs savoirs et savoir-faire sur le terrain sans cette explicitation, qui est donc loin d'être strictement nécessaire. Cette explicitation peut être toutefois utile, notamment lorsqu'il s'agit des moments d'institutionnalisation (Brousseau, 1998): faire prendre conscience de ce qui, dorénavant, fera référence, demande une explicitation. L'explicitation des prises d'information peut également être utile lors des moments de guidage par le formateur : par exemple, comme nous l'avons évoqué précédemment, exprimer que les Bois Raméaux Fragmentés doivent être "non défibrés " est plus porteur de sens que d'indiquer qu'ils doivent être "beaux ". Cette explicitation, à notre sens, n'est pas incompatible avec une phase de recherche par les apprenants qui peuvent être amenés à commencer à construire par eux-mêmes une représentation de la situation professionnelle. Suite à Ochanine (1978), nous pensons que toute la difficulté pour les formateurs consiste à résoudre la tension entre :

- la possibilité pour l'apprenant d'être confronté à la situation professionnelle dans son ensemble, avec diverses possibilités de prendre des informations, et de trouver par luimême les informations pertinentes et celles qui, au moins momentanément, peuvent être laissées de côté ;

- un dispositif strict pour guider les prises d'information; par exemple, Ochanine (Idem) montre que pour l'apprentissage de l'anatomie, un dessin au trait, ne mettant en évidence que les caractéristiques pertinentes et servant ainsi de guidage perceptif, est plus efficace qu'une photographie, qui offre une multitude de prises d'information possible, dont toutes ne sont pas pertinentes. 


\section{Conclusion}

Cet article souhaitait articuler deux recherches dans des contextes différents : agricole et industriel. Dans ces deux contextes, nous avons observé deux manières de conduire l'activité :

- une première manière que nous qualifions de conventionnelle, c'est-à-dire faiblement mécanisée pour la technique BRF et sur des machines outils standard dans l'industrie.

- une seconde manière que nous qualifions d'instrumentée va par exemple utiliser le tracteur pour le transport du broyat et un outil d'épandage par la suite ; dans l'industrie, c'est la commande numérique qui va instrumenter l'action.

Cette catégorisation montre que les prises d'information dans chaque manière de conduire l'activité vont s'avérer pertinentes pour lire l'activité professionnelle et l'activité de formation professionnelle.

Il n'en demeure pas moins que, dans un but de formation, se pose la question du niveau de conscience des professionnels lors des prises d'information, et donc de l'accès à l'activité cognitive. Face aux diverses possibilités de prises d'information, il nous semble important de comprendre ce qui conditionne ces choix, ce qui va générer la pertinence d'une information. Les représentations pour l'action sont à l'œuvre car elles participent à l'appropriation de la situation, et par un chemin inverse la manière d'intégrer la situation va agir et travailler la représentation pour l'action. Ce double mouvement doit pouvoir être mis au jour et articulé, tel était le sens de notre démarche. Dans tous les cas, c'est selon nous une perspective à développer pour concevoir des dispositifs de formation susceptibles de favoriser la construction de compétences professionnelles. 


\section{Bibliographie}

Beauvois, J.-L., \& Dubois, N. (1991). Internal/external orientations and psychological information processing. Cahiers de Psychologie Cognitive, European Bulletin of Cognitive Psychology, 11(2), 193-212.

Becerril, R. (2008). Contexte professionnel, contexte de la formation technologique supérieure, approche didactique. Le cas des formations utilisant des simulateurs informatiques. Thèse en sciences de l'éducation. Non publié. Université Paul Sabatier, Toulouse III.

Bertrand, L., \& Weill-Fassina, A. (1993). Formes de représentations fonctionnelles et contrôle des actions dans le diagnostic de panne. In A. Weill-Fassina, P. Rabardel \& D. Dubois (Eds.), Représentations pour l'action (pp. 247-269). Toulouse: Octarès.

Brousseau, G. (1998). Théorie des situations didactiques, textes rassemblés par Balacheff $N$. et al. Grenoble: La Pensée Sauvage.

Carter, K., Cushing, K., Sabers, D., Stein, R., \& Berliner, D. (1988). Expert-novice differences in perceiving and processing visual classroom information. Journal of Teacher Education, 39(3), 25-31.

Chase, W. G., \& Simon, H. A. (1973). Perception in chess. Cognitive Psychology, 4, 55-81.

Desnoyers, L. (2004). La prise d'information. Récepteurs et fureteurs. In P. Falzon (Ed.), Ergonomie (pp. 83-98). Paris: PUF.

Enard, C. (1968). Etude des informations permanentes : infrastructure géographique des secteurs et carte mentale du contrôleur. Rocquencourt: INRIA.

Fabre, I., \& Veyrac, H. (2008). Des représentations croisées pour l'émergence d'une médiation de l'espace documentaire. Communication \& Langages, 156, 103-115.

Fraysse, B., \& Murillo, A. (2010). Enseigner des pratiques culturales émergentes : un exemple de partenariat multi-acteurs. XVI Congreso Mundial de Ciencias de la Educacion, Monterrey.

Lave, J. (1988). Cognition in Practice. Mind, Mathematics and Culture in Everyday Life. Cambridge: Cambridge University Press.

Leplat, J. (1985). Les représentations fonctionnelles dans le travail. Psychologie Française, 30(3/4), 269-275.

Leplat, J., \& Hoc, J.-M. (1983). Tâche et activité dans l'analyse psychologique des situations. Cahiers de Psychologie Cognitive, 3(1), 49-63.

Maurice, J.-J. (2006). L'expérience de l'enseignant : une réflexivité limitée. Formation et pratiques d'enseignement en questions, 3, 53-67.

Murillo, A. (2010). Le niveau de difficulté des tâches scolaires : des marges de manœuvre limitées pour les enseignants. Carrefours de l'Education, 29, 79-93.

Murillo, A. (2010). Recherche-Formation sur les Bois Raméaux Fragmentés, rapport de postdoctorat. Non publié. Ecole Nationale de Formation Agronomique.

Ochanine, D. (1978). Le rôle des images opératives dans la régulation des activités de travail. Psychologie et Education, I(2), 63-72.

Pastré, P. (2002). L'analyse du travail en didactique professionnelle. Revue Française de Pédagogie, 138, 9-17.

Pastré, P., Mayen, P., \& Vergnaud, G. (2006). La didactique professionnelle. Revue Française de Pédagogie, 154, 145-198.

Rabardel, P. (1993). Micro-genèse et fonctionnalité des représentations dans une activité avec instrument. In A. Weill-Fassina, P. Rabardel \& D. Dubois (Eds.), Représentations pour l'action (pp. 113-137). Toulouse: Octarès. 
Rabardel, P. (1995). Les hommes et les technologies. Approche cognitive des instruments contemporains. Paris: Armand Colin.

Rabardel, P. (2005). Instrument subjectif et développement du pouvoir d'agir. In P. Rabardel \& P. Pastré (Eds.), Modèles du sujet pour la conception (pp. 11-29). Toulouse: Octarès.

Rabardel, P., Carlin, N., Chesnais, M., Lang, N., \& Pascal, M. (1998). Ergonomie: concepts et méthodes. Toulouse: Octarès.

Rabardel, P., Rak, I. \& Vérillon, P. (1988). Machines outils à commande numérique, approches didactiques. Rapport de recherche: INRP.

Rogalski, J. \& Samurçay, R. (1994). Modélisation d'un « savoir de référence " et transposition didactique dans la formation de professionnels de haut niveau. In G. Arsac, Y. Chevallard, J-L. Martinand \& A. Tiberghien (Ed). La transposition didactique à l'épreuve. Grenoble : La Pensée Sauvage. p. 35-72.

Samurçay, R., \& Hoc, J.-M. (1988). De l'analyse du travail à la spécification d'aides à la décision dans des environnements dynamiques. Psychologie Française, 33(3), 187196.

Savoyant, A. (2008). Quelques réflexions sur les savoirs implicites. Travail et apprentissages, 1, 92-100.

Teiger, C. (1993). Représentation du travail et travail de la représentation. In A. WeillFassina, P. Rabardel \& D. Dubois (Eds.), Représentations pour l'action (pp. 187-200). Toulouse: Octarès.

Thuilliez, H. (2005). Rôle de l'opérateur et du statut fonctionnel des informations lors de la prise d'informations en anesthésie. Le travail humain, 68(3), 225-252.

Vergnaud, G. (1985). Concepts et schèmes dans une théorie opératoire de la représentation. Psychologie française, 254-251.

Vermersch, P. (1981). Image opérative ou représentation fonctionnelle ? In L'image opérative. Actes d'un séminaire et recueil d'articles de D. Ochanine. Paris.

Weill-Fassina, A., Rabardel, P., \& Dubois, D. (1993). Représentations pour l'action. Toulouse: Octarès Editions. 